\title{
Radical copolymerization of styrene and acrylonitrile in the presence of oligooxyethylene azoester
}

\author{
Ewa Wiśniewska, ${ }^{*}$ Barbara Pabin-Szafko
}

Szczecin University of Technology, Polymer Institute, 10, Pułaski St., 70-322 Szczecin, Poland; fax: +48 91 4494685; email: ewaw@ps.pl

(Received: 27 July, 2007; published:13 November, 2007)

\begin{abstract}
Telechelic vinyl oligomers terminated with hydroxyl groups were prepared by free radical copolymerization of styrene (S) and acrylonitrile (AN), initiated by azoester-2,2'-azobis[2-methyl- $\omega$-hydroxy-oligo(oxyethylene) propionate] [AIB-OOE $(400)]$, carried out in $N, N$-dimethylformamide. The reactivity ratios $r_{S}=0,225$ and $r_{A N}=0,722$ for styrene and acrylonitrile, respectively, were evaluated by Kelen-Tüdős method. The glass transition temperatures $\left(T_{g}\right)$ of the copolymers were determined by differential scanning calorimetry. $T_{g}$ values varied with the copolymer composition in the range of $60-82{ }^{\circ} \mathrm{C}$ and the maximum $\mathrm{T}_{9}$ value was obtained for copolymer with $60 \mathrm{~mol} \%$ AN.
\end{abstract}

\section{Introduction}

The process of radical copolymerization of styrene and acrylonitrile has been the subject of many investigations [1-9]. The copolymerizations were carried out at different environments, and the kinetics and mechanism of polyreactions, as well as the structure and properties of obtained copolymers have been studied [1-3]. The investigators view relating to the mechanism of styrene (S) - acrylonitrile (AN) copolymerization is divided on this issue. Some of them claim that this copolymerization is a marked example of the occurrence of penultimate unit effect [4, 5], the others claim that the terminal model also adequately describes the reaction mechanism [6-8]. Determined reactivity ratios of the comonomers ranged from 0.04 to 0.34 for acrylonitrile and from 0.14 to 0.54 for styrene [10]. They are differentiated depending on the reaction conditions and methods of their evaluation. The SAN copolymers were prepared mainly by the copolymerization initiated by smallmolecule initiators: 2,2'-azobisisobutyronirile (AIBN) or benzoyl peroxide (BPO) [11].

It seems interesting to examine the possibility of preparation of the copolymers with the application of the functional initiators - macro-azoinitiators, whose molecules are terminated with e.g. hydroxyl groups. Particularly in the case of vinyl monomers, such as styrene or acrylonitrile, when the reaction of chain termination proceeds via the macroradicals recombination [12-14], the telechelic copolymers can be the products of the reaction $[15,16]$.

The objective of this work was to prepare styrene-acrylonitrile oligomers in the presence of oligomeric functional initiator - 2,2'-azobis[2-methyl-w-hydroxyoligo(oxyethylene) propionate] [AIB-OOE(400)], in which the oligooxyethylene chains with hydroxyl functional groups are significant fractions of the macromolecules [17]. Such copolymers, termed as "block-copolymerlike", can be utilized as prepolymers 
for the subsequent polyreactions, resulting in the modification of the properties of the final products.

\section{Results and discussion}

The kind and chemical structure of the initiator molecule have a influence on the course of radical copolymerization and the mutual reactivity of the comonomers. In order to estimate the monomer reactivity ratios, the compositions of the oligovinyl fragment in S-AN copolymers were determined on the basis of absorption measurements $\left(A_{S}\right.$ and $\left.A_{A N}\right)$ for the bands at established wave numbers by means of the base line method using a previously determined calibration curve $A_{S} / A_{A N}=$ $f([S] /[A N])$, for the mixtures of styrene and acrylonitrile homopolymers. Subsequently the instantaneous copolymer composition was obtained by measurement of the ratio of these absorbing bands in the copolymers.

The values of the monomer reactivity ratios $\left(r_{S}\right.$ and $r_{A N}$ for styrene and acrylonitrile, respectively) were calculated according to the Kelen-Tüdös method by the composition equation [18]:

$\eta=\left(r_{S}+\frac{r_{A N}}{\alpha}\right) \cdot \xi-\frac{r_{A N}}{\alpha}$

where $\eta$ and $\xi$ are variables defined as:

$$
\eta=\frac{x(y-1)}{\alpha y+x^{2}} ; \xi=\frac{x^{2}}{\alpha y+x^{2}}
$$

whereas $\alpha$ is a auxiliary parameter:

$$
\alpha=\frac{x_{\min } \cdot x_{\max }}{\sqrt{y_{\min } \cdot y_{\max }}}
$$

$x$ denotes the initial composition of the monomers in the reaction mixture $\left([\mathrm{S}]_{0} /[\mathrm{AN}]_{0}\right)$, $y$ is the composition of the copolymer expressed in terms of the molar fractions of monomer units in the copolymer $(\mathrm{d}[\mathrm{S}] / \mathrm{d}[\mathrm{AN}])$.

Equation (1), with such defined variables $\eta$ and $\xi$, serves for determination of the reactivity ratios at low conversion of the monomers. However, the parameter $z$ defined by Equation (4) should be taken into account at high conversion (extended Kelen-Tüdős method) [19-21]:

$$
z=\frac{\lg \frac{[S]}{[S]_{0}}}{\lg \frac{[A N]}{[A N]_{0}}}=\frac{\lg \left(1-\zeta_{S}\right)}{\lg \left(1-\zeta_{A N}\right)}
$$

where $\zeta_{\mathrm{S}}$ and $\zeta_{\mathrm{AN}}$ are the partial conversions of particular monomers:

$$
\zeta_{A N}=w \frac{\mu+x_{0}}{\mu+y} \quad \text { and } \quad \zeta_{S}=\frac{y}{x_{0}} \zeta_{A N}
$$

in which $w$ designates a weight conversion, $\mu=\mu_{\mathrm{AN}} / \mu_{\mathrm{S}}$ - ratio of the molecular weights of monomers - acrylonitrile and styrene, respectively, $x_{0}$ - initial composition of the monomers mixture. 
Authors of the above method, Kelen and Tüdős, have also defined an average value of the monomers composition $\bar{x}$, by the $y$ and $z$ values as consistent with the current conversion:

$\bar{x}=\frac{y}{z}$

the application of which in Equation (1) instead of $x$, through the calculation of variables $\eta$ and $\xi$, expressed as follows:

$\eta=\frac{z(y-1)}{\alpha z^{2}+y} \quad$ and $\quad \xi=\frac{y}{\alpha z^{2}+y}$

allowed the determination of the reactivity ratios $r_{S}$ and $r_{A N}$ in the investigated process (Fig. 1). Because it is a graphical method, correctness of the assumed mechanism can be immediately ascertained by the linearity of the points in the plot $\eta$ $=f(\xi)$.

Tab. 1. Composition of the reaction mixture $\left([M]_{0}=2.5 \mathrm{~mol} \cdot \mathrm{dm}^{-3},[\mathrm{l}]_{0}=0.04 \mathrm{~mol} \cdot \mathrm{dm}^{-3}\right)$ and characteristics of copolymers.

\begin{tabular}{cccccc}
\hline Copolymer & $\begin{array}{c}{[\mathrm{S}]_{0} /[\mathrm{AN}]_{0}{ }^{\text {a) }}} \\
\left(\mathrm{mol}^{-1} \mathrm{~mol}^{-1}\right)\end{array}$ & $\begin{array}{c}\mathrm{d}[\mathrm{S}] / \mathrm{d}[\mathrm{AN}]^{\mathrm{b})} \\
\left(\mathrm{mol}^{\mathrm{b}} \cdot \mathrm{mol}^{-1}\right)\end{array}$ & $\begin{array}{c}\text { Conversion } \\
\left(\mathrm{wt}^{\%}\right)\end{array}$ & $\begin{array}{c}\bar{M}_{n} \\
\left(\mathrm{~g} \cdot \mathrm{mol}^{-1}\right)\end{array}$ & $\begin{array}{c}T_{g} \\
\left({ }^{\circ} \mathrm{C}\right)\end{array}$ \\
\hline SAN 1 & 9.081 & 4.102 & 33.0 & 3600 & 63.6 \\
SAN 2 & 4.000 & 4.040 & 33.1 & 3800 & 58.6 \\
SAN 3 & 2.305 & 1.318 & 46.6 & 3720 & 74.3 \\
SAN 4 & 1.500 & 1.010 & 45.9 & 5000 & 75.7 \\
SAN 5 & 1.000 & 0.732 & 41.6 & 7200 & 81.4 \\
SAN 6 & 0.668 & 0.555 & 47.6 & 4500 & 80.0 \\
SAN 7 & 0.426 & 0.430 & 48.5 & 5000 & 82.0 \\
SAN 8 & 0.110 & 0.233 & 17.0 & 4800 & 71.9 \\
a) initial ratio of monomers in the reaction mixture $\left(x_{0}\right)$ \\
b) ratio of monomer units in copolymer $(y)$
\end{tabular}

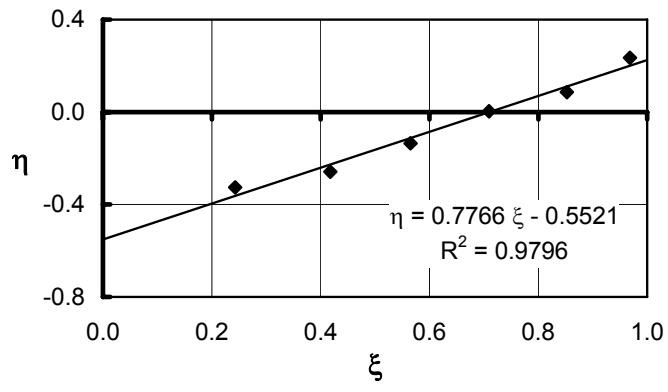

Fig. 1. Kelen-Tüdős plot for determination of the reactivity ratios in the copolymerization of acrylonitrile and styrene.

The values of reactivity ratios $r_{S}=0.225$ and $r_{A N}=0.722$ were determined and the rectilinear dependence $\eta=\mathrm{f}(\xi)$ (Fig. 1) confirms the rightness of the assumed calculation method (according to the terminal model). The obtained results are associated with the presence of azoester AIB-OOE $(400)$ in the reaction system. The oligoether chains of the AIB-OOE $(400)$ can be the matrix ordering the AN monomer 
and by this means they accelerate the acrylonitrile addition both to the macroradical ended with the same unit (causing a growth of $r_{A N}$ ), and the styrene unit (decreasing value of $r_{S}$ ).

On the basis of the changes in the reactivity ratios of comonomers depending on the copolymerization environment, one can expect the changes in the sequences distribution in the copolymer. Moreover, knowledge of the reactivity ratio values under the specified conditions enables such a design of the monomers mixture composition in order to obtain the copolymer with appropriate composition and properties (Fig. 2, Table 1).

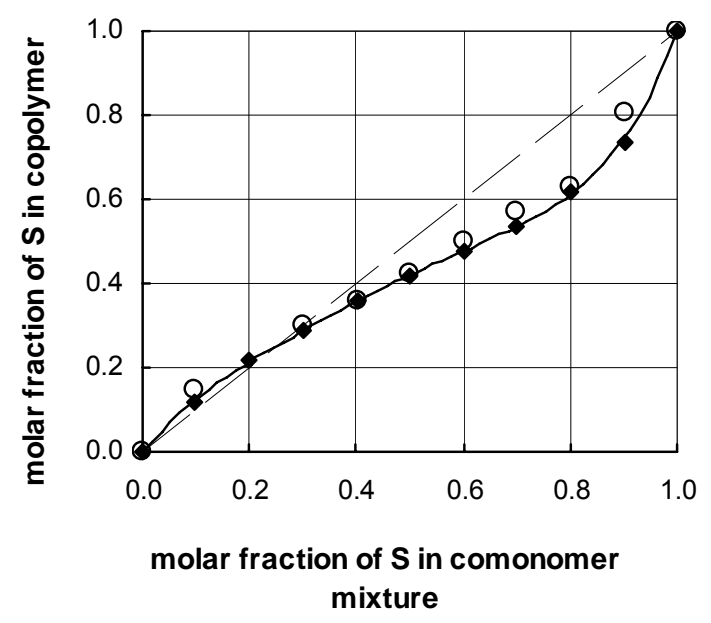

Fig. 2. Dependence of the copolymer composition on the composition of monomers mixture: - points evaluated for established values of $r_{S}=0.225$ and $r_{A N}=0.722 ; \circ-$ experimental points

As can be seen (Table 1), the small values of the copolymer molecular weights testify about the oligomeric products of the syntheses. Moreover the presence of the oligomeric azoester AIB-OOE(400) (as reaction initiator) causes the oligoether chains to be the significant fraction in the copolymer macromolecules. The influence of oligomers composition on their important characteristics - thermal properties [22], was investigated in the temperature range of $-50-280{ }^{\circ} \mathrm{C}$, by the determination of the glass transition temperatures $T_{g}$ (Table 1). A single $T_{g}$ was observed, the value of which increases along with increasing $A N$ fraction in the copolymer. The maximum value $\left(81,4-82,0^{\circ} \mathrm{C}\right)$ was achieved at the molar composition $\sim 60 \mathrm{~mol} \%$ AN in the copolymer, and then this temperature decreases to $\sim 72^{\circ} \mathrm{C}$ at $80 \mathrm{~mol} \%$ AN (Fig. 3).

The results reported in work, for example [9], indicate, that $T_{g}$ dependence on the composition of SAN copolymer changes in the temperature range $105-117^{\circ} \mathrm{C}$, and reaches a maximum at 50 wt\% content of styrene. In the case of PS the $T_{g}$ value amounts to $102{ }^{\circ} \mathrm{C}$ and for PAN $-94^{\circ} \mathrm{C}$.

The decrease of $T_{g}$ value in the examined ether-acrylonitrile-styrene copolymers, results mainly from the presence of the oligoether chains derived from the initiator AIB-OOE (400) which causes the increase of mobility of the oligomeric chains, as well as due to low molecular weights of the copolymers. 


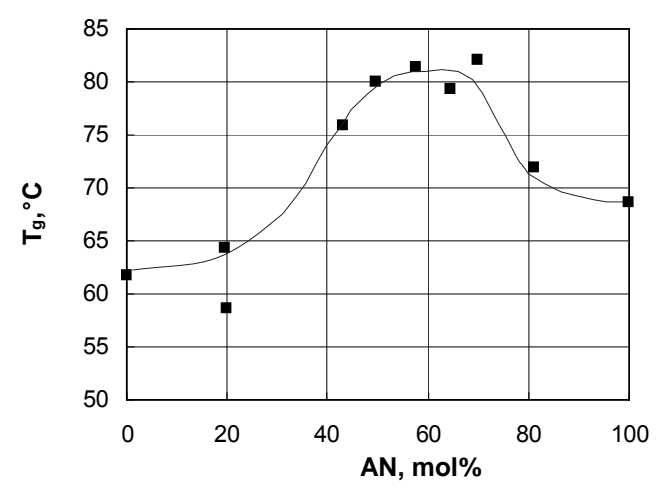

Fig. 3. Dependence of $T_{g}$ on the composition of styrene-acrylonitrile segment of copolymer macromolecules.

\section{Conclusions}

The application of telechelic, oligomeric azoinitiator AIB-OOE (400) in the copolymerization of styrene and acrylonitrile enables the preparation of essentially a three-component product, with a low molecular weight. The products can be utilised for the subsequent polyreactions as prepolymers having the functional groups or as effective compatibilizers in various immiscible polymer systems, simultaneously affecting the change of properties, e.g. an improvement of resistance on solvents or oils.

The AIB-OOE (400) initiator causes the increase of the reactivity ratio of acrylonitrile in the copolymerization with styrene, and the acceleration of the polymerization reaction of acrylonitrile, this being the subject of work published in [23].

\section{Experimental part}

The monomers: styrene and acrylonitrile were purified by washing several times with $5 \%$ aqueous $\mathrm{NaOH}$ solution to remove inhibitors. Subsequently the monomers were neutralised by washing with distilled water, dried over anhydrous $\mathrm{MgSO}_{4}$ and distilled in $\mathrm{N}_{2}$ under reduced pressure in the presence of hydroquinone and $\mathrm{CaH}_{2}$. Solvent $\mathrm{N}, \mathrm{N}$-dimethylformamide (DMF) was purified by rectification carried out at normal pressure. Azoinitiator AIB-OOE $(400)$ with $\bar{M}_{n}=1100 \mathrm{~g} \cdot \mathrm{mol}^{-1}$ was prepared by means of modified Pinner's method from AIBN and oligo(oxyethylenediol) with $\bar{M}_{n}=$ $400 \mathrm{~g} \cdot \mathrm{mol}^{-1}[17]$ :

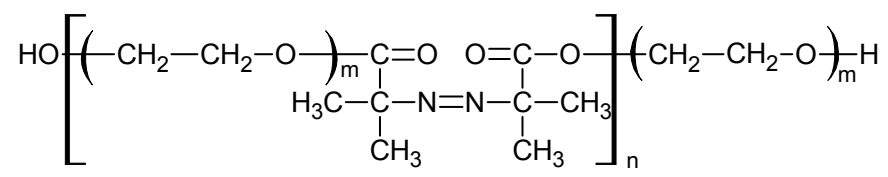

AIB-OOE $(400)$

$(\mathrm{m} \approx 8.8 ; \mathrm{n} \approx 1.1)$

The copolymerization reaction of styrene and acrylonitrile was carried out in solution at $60{ }^{\circ} \mathrm{C}$ under $\mathrm{N}_{2}$ atmosphere using AIB-OOE (400) as a functional azoinitiator and DMF as a solvent. The overall concentration of the monomers was constant $\left([M]_{0}=\right.$ $2.5 \mathrm{~mol} \cdot \mathrm{dm}^{-3}$ ) and the initiator concentration of []$_{0}=0.04 \mathrm{~mol} \cdot \mathrm{dm}^{-3}$ was selected for all experiments. The molar fractions of the monomers in the reaction mixture were 
varied in the consecutive syntheses by increasing acrylonitrile fraction. After the reaction had proceeded $(360 \mathrm{~min})$ the copolymers were precipitated in methanol/water (90/10 V/V) mixture, purified by redissolution in chloroform, followed by the precipitation and then dried at $40{ }^{\circ} \mathrm{C}$ under vacuum to a constant mass.

The copolymer compositions were determined by IR spectroscopy (Specord M80 with application of software) utilising the characteristic absorption bands for styrene $\left(3028 \mathrm{~cm}^{-1}\right)$ and acrylonitrile $\left(2240 \mathrm{~cm}^{-1}\right)$ monomer units. The molecular weights of copolymers were determined by vapour pressure osmometry (VPO Knauer, operating range of molecular weights: $40-35000 \mathrm{~g} \cdot \mathrm{mol}^{-1}$ ), in DMF as a solvent at temperature of $100{ }^{\circ} \mathrm{C}$. The thermal properties were examined by differential scanning calorimetry (DSC 910 - TA Instruments) under $\mathrm{N}_{2}$ atmosphere at a heating rate of $10^{\circ} \mathrm{C} \cdot \mathrm{min}^{-1}$.

\section{References}

[1] Aldeeb, A. A.; Rogers, W. J.; Mannan, M. S. J. Hazardous Materials 2003, 104, 269.

[2] Pietrasik, J.; Dong, H.; Matyjaszewski, K. Macromolecules 2006, 39, 6384.

[3] Połowiński, S. Prog. Polym. Sci. 2002, 27, 537.

[4] Heuts, J. P. A.; Gilbert, R. G.; Maxwell, I. A. Macromolecules 1997, 30, 726.

[5] Hill, D. J. T.; O'Donnell, J. H.; O'Sullivan, P. W. Macromolecules 1982, 15, 960.

[6] Pichot, Ch.; Pham, Q-T. Makromol. Chem. 1979, 180, 2359.

[7] Brar, A. S.; Hekmatyar, S. K. J. Polym. Sci.: Part A: Polym. Chem. 1999, 37, 721.

[8] Bag, D. S.; Maiti, S. J. Polym. Sci.: Part A: Polym. Physics. 1997, 35, 2049.

[9] Li, D.; et al. J. Thermal Analysis and Calorimetry 2001, 63, 69.

[10] Brandrup, J.; Immergut, E. H. "Polymer Handbook", $3^{\text {rd }}$ Ed. John Wiley \& Sons Inc., 1989.

[11] Sandler, S. R.; Karo, W. Polymer Syntheses, vol. 1, $2^{\text {nd }}$ Ed. Acad. Press Inc., 1992.

[12] Bamford, C. H.; et al. "The Kinetics of Vinyl Polymerization by Radical Mechanisms", Butterworths Sci. Publ. London, 1958.

[13] Moad, S.; Solomon, D. H. "The Chemistry of Radical Polymerization", Pergamon, Elsevier Sci. Ltd., 1995.

[14] Matyjaszewski, K.; Davis, T. P. "Handbook of Radical Polymerization", WileyIntersci., 2002.

[15] Furukawa, J.; Takamori, S. Yamashita, S. Angew. Makromol. Chem. 1967, 1, 92.

[16] Oppenheimer, C.; Heitz, W. Angew. Makromol. Chem. 1981, 98, 167.

[17] Pabin-Szafko, B.; Wiśniewska, E.; Szafko, J. Polimery 2005, 50, 271.

[18] Kelen, T.; Tüdős, F. React. Kinetics and Catalysis Lett. 1974, 1, 487.

[19] Tüdős, F.; Kelen, T.; Földes-Berezsnich, T.; Turcsányi, B. J. Macromol. SciChem. 1976, A10(8), 1513.

[20] Mahdavian A. R. , Abdollahi M., Mokhtabad L., Bijanzadeh H. R., Ziaee F. J. Appl. Polym. Sci. 2006, 101, 2062.

[21] Semsarzadeh M. A., Daronkola M. R. R., Abdollahi M. J. Macromol. Sci., Part A: Pure Appl. Chem. 2007, 44, 953.

[21] Turi, E. A. "Thermal characterization of polymeric materials", $2^{\text {nd }}$ Ed. Acad. Press, 1997.

[22] Wiśniewska, E.; Pabin-Szafko, B. Polimery 2008, 52, no 1 (will be published). 